\title{
Follicular Lymphoma by Ann Arbor Stage
}

National Cancer Institute

\section{Source}

National Cancer Institute. Follicular Lymphoma by Ann Arbor Stage. NCI Thesaurus. Code C141255.

An anatomic stage for follicular lymphoma based on the Ann Arbor classification criteria. 